\title{
Assessment of Spatiotemporal Fusion Algorithms for Planet and Worldview Images
}

\author{
Chiman Kwan ${ }^{1, *} \mathbb{1}$, Xiaolin Zhu ${ }^{2}$, Feng Gao ${ }^{3}$, Bryan Chou ${ }^{1}$, Daniel Perez ${ }^{4}{ }^{(\mathbb{D})}$, Jiang Li ${ }^{4}$, \\ Yuzhong Shen ${ }^{4}$, Krzysztof Koperski ${ }^{5}$ and Giovanni Marchisio ${ }^{5}$ \\ 1 Applied Research LLC, Rockville, MD 20850, USA; choub90@gmail.com \\ 2 Department of Land Surveying and Geo-Informatics, The Hong Kong Polytechnic University, \\ Kowloon, Hong Kong 999077, China; xiaolin.zhu@polyu.edu.hk \\ 3 Hydrology \& Remote Sensing Lab, USDA ARS, Beltsville, MD 20704, USA; feng.gao@ars.usda.gov \\ 4 Department of Electrical \& Computer Engineering, Old Dominion University, Norfolk, VA 23529, USA; \\ dpere013@odu.edu (D.P.); jli@odu.edu (J.L.); yshen@odu.edu (Y.S.) \\ 5 Digital Globe, Inc., Herndon, VA 20171, USA; kkopersk@digitalglobe.com (K.K.); \\ giovanni.marchisio@comcast.net (G.M.) \\ * Correspondence: chiman.kwan@signalpro.net; Tel.: +1-240-207-2311
}

Received: 7 March 2018; Accepted: 30 March 2018; Published: 31 March 2018

\begin{abstract}
Although Worldview-2 (WV) images (non-pansharpened) have 2-m resolution, the re-visit times for the same areas may be seven days or more. In contrast, Planet images are collected using small satellites that can cover the whole Earth almost daily. However, the resolution of Planet images is $3.125 \mathrm{~m}$. It would be ideal to fuse these two satellites images to generate high spatial resolution $(2 \mathrm{~m})$ and high temporal resolution (1 or 2 days) images for applications such as damage assessment, border monitoring, etc. that require quick decisions. In this paper, we evaluate three approaches to fusing Worldview (WV) and Planet images. These approaches are known as Spatial and Temporal Adaptive Reflectance Fusion Model (STARFM), Flexible Spatiotemporal Data Fusion (FSDAF), and Hybrid Color Mapping (HCM), which have been applied to the fusion of MODIS and Landsat images in recent years. Experimental results using actual Planet and Worldview images demonstrated that the three aforementioned approaches have comparable performance and can all generate high quality prediction images.
\end{abstract}

Keywords: image fusion; Planet; Worldview; pansharpening; forward prediction; spatiotemporal

\section{Introduction}

Due to high spatial resolution, Worldview-2 (WV) images have been widely used for hazard assessment, nuclear site monitoring, border activity detection, etc. One issue is the revisit time of WV satellite. On the other hand, although the resolution of Planet images is lower than that of WV, Planet images use multiple small satellites to collect images and can cover the same area almost daily. It would be ideal to fuse the above images to create a high spatial and high temporal resolution image sequence so that quick and responsive actions can be taken for certain applications such as damage assessment due to hurricanes, fires, tsunamis, etc.

One may question the necessity of this research, as the spatial resolutions of WV (2 m) and Planet $(3.125 \mathrm{~m})$ images are all of high resolution and do not seem to differ that much. A simple bicubic interpolation of Planet images should be sufficient. It turns out that, as can be seen in Sections 2 and 3, the visual appearance of Planet images seems to be much worse than $3.125 \mathrm{~m}$. We interacted with Planet engineers and inquired about why the Planet images do not seem to have $3.125 \mathrm{~m}$ resolution, but we could not get any direct feedback. We speculated that there might be some smearing effects due to various point spread functions [1,2] in the imaging hardware in Planet satellites. 
It is also important to emphasize that the goal of our research is very different from traditional remote sensing applications such as vegetation monitoring, forest monitoring, etc. Our goal is to perform border monitoring, which includes monitoring of buildings, military built-up, and road construction near borders, illegal trail detection, illegal tunnel digging activity detection, etc., using high resolution satellite images. Conventional remote sensing platforms such as Landsat (30 m resolution, 16-day revisit time) and MODIS (500 $\mathrm{m}$ resolution and almost daily revisit) do not have enough spatial resolution to meet our sponsor's needs. Therefore, we have looked at Worldview and Planet images. After some investigations, we found that the Planet images' resolution is not as good as it should be. Consequently, we decided to embark on this research effort and see if we could generate high temporal and high spatial resolution images by fusing Worldview and Planet images.

There have been on-going research activities in fusing MODIS and Landsat images throughout the past decade. In [3], a fusion approach known as Spatial and Temporal Adaptive Reflectance Fusion Model (STARFM) was proposed and demonstrated. Several alternative algorithms [4-6] were published to further improve the fusion performance. According to a review paper [7], the Spatial Temporal Adaptive Algorithm for mapping Reflectance Change (STAARCH) [4] approach can handle abrupt changes, but requires two pairs of MODIS and Landsat images. The enhanced spatial and temporal adaptive reflectance fusion model (ESTARFM) [5] algorithm focuses on enhancing performance of mixed pixels. Similar to STAARCH, ESTARFM requires two pairs of images. As a result, both STAARCH and ESTARFM may not be suitable for forward prediction, i.e., using the images in the past to predict the image at the future time. Recently, two approaches have been proposed for fusing MODIS and Landsat. In [8], Flexible Spatiotemporal Data Fusion (FSDAF) was proposed, which can handle heterogeneous images very well. In [9], the Hybrid Color Mapping (HCM) approach was proposed for fusing MODIS and Landsat images and the idea works well for homogeneous images. In addition to the above papers, there are other fusion approaches in the literatures. See [10-15] and references therein.

Pansharpening refers to the use of a high resolution panchromatic (pan) band to sharpen low resolution multispectral bands. Many approaches have been proposed in the past two decades. In recent years, new pansharpening approaches have been developed that can utilize high resolution multispectral bands for pansharpening. See [16,17] and references therein. After a careful study of the fusion problem between WV and Planet, it turns out that pansharpening algorithms, such as the algorithms mentioned in [16,17], cannot be applied. The main reason is that most existing pansharpening algorithms require low resolution multi-spectral images and a high resolution pan image at the time of prediction. In the fusion of WV and Plant images, this is not possible. We have actually applied several pansharpening algorithms such as Gram-Schmidt Adaptive (GSA), Principal Component Analysis (PCA), etc. in [16] to enhance a MODIS image at time $t_{2}$ by using a better resolution Landsat image at an earlier time $t_{1}$. The results are not satisfactory due to severe changes in image contents between the two images.

In this paper, we summarize the results of applying STARFM, FSDAF, and HCM approaches to fusing WV and Planet images for forward prediction. To the best of our knowledge, no one has carried out such a study before for WV and Planet images. The first two approaches, STARFM and FSDAF, are well-known in fusing the MODIS and Landsat community. The third approach (HCM) was motivated by our recent pansharpening work for synthesizing a high resolution hyperspectral image by fusing a high resolution color image with a low resolution hyperspectral image cube [18]. Similar to STARFM and FSDAF, only one pair of Planet and WV images is needed for prediction. Experiments using actual Planet and WV images were used to quantify the performance of the three approaches.

Our paper is organized as follows. Section 2 briefly reviews the three fusion methods. Section 3 presents our experimental results. Finally, conclusions and future research directions will be presented in Section 4. 


\section{Spatiotemporal Fusion Approaches}

In the next few sections, we will briefly summarize the STARFM, FSDAF, and HCM algorithms. Both Planet and WV images are co-registered and resampled to the same image size and extent according to the requirement of these fusion algorithms.

\subsection{STARFM}

If ground cover type and system errors at pixel $(x, y)$ have not changed between the observation date $t_{k}$ and the prediction date $t_{p}$, where a WV scene is not available, then the temporal changes of surface reflectance from WV should be equivalent to the temporal changes in the corresponding Planet pixel between two dates. If the bias between WV and Planet stays the same for two dates $\left(\varepsilon_{p}=\varepsilon_{k}\right)$, we have

$$
W\left(x, y, t_{p}\right)=W\left(x, y, t_{k}\right)+\left(P\left(x, y, t_{p}\right)-P\left(x, y, t_{k}\right)\right)
$$

where $W, P$ denote pixels in $W V$ and Planet images, respectively.

To address mixed pixels in the prediction, STARFM $[3,7]$ introduces additional information from neighboring pixels and uses spectrally similar pixels for prediction. That is, the predicted surface reflectance for the central pixel at date $t_{p}$ is computed with a weighting function from spectrally similar neighboring pixels within a specified search window:

$$
W\left(w / 2, w / 2, t_{p}\right)=\sum_{k=1}^{M} \sum_{i=1}^{N} \alpha_{i k} \times\left(W\left(x, y, t_{k}\right)+\left(P\left(x, y, t_{p}\right)-P\left(x, y, t_{k}\right)\right)\right)
$$

where $w$ is the searching window size and $(w / 2, w / 2)$ is the central pixel of this moving window. The size of the searching window was determined as three coarse resolution pixels. In this paper, the size of the searching window is defined as $12 \mathrm{~m}$ ( $\pm 6 \mathrm{~m}$ searching distance), which covers about three Planet pixels. $N$ represents the total number of similar Planet pixels in the moving window and $i$ is the index. $M$ represents the total number of pairs of observed Planet and WV images. The weighting function $\alpha_{i k}$ determines how much each neighboring pixel, $i$, contributes to the estimated reflectance of the central pixel from image pair $k$. Mathematically the weighting $\alpha_{i k}$ is given by

$$
\begin{gathered}
\alpha_{i k}=\left(1.0 / C_{i k}\right) / \sum_{i=1}^{N}\left(1.0 / C_{i k}\right) \\
C_{i k}=\left|P\left(x, y, t_{k}\right)-P\left(x, y, t_{p}\right)\right| *\left|P\left(x, y, t_{k}\right)-W\left(x, y, t_{k}\right)\right| * D_{i}
\end{gathered}
$$

where $D_{i}$ is the distance between candidate pixel $(i)$ at location $(x, y)$ and the central pixel of the moving window at location $(w / 2, w / 2)$. It is determined by three measures based on: (1) spectral difference between the Planet and WV data at a given location; (2) temporal difference between the input Planet data $\left(P\left(x, y, t_{k}\right)\right)$ and the Planet data at the prediction date $\left(P\left(x, y, t_{p}\right)\right)$; and (3) the geographic distance between the central pixel and the candidate pixel. Closer pixels are weighted more. Pixels with smaller spectral and temporal differences also receive higher weight. Weights for all spectrally similar pixels are normalized (so the sum of weights equals one) before applying to Equation (2). These measures ensure that pure and close neighbor pixels get higher weights in the prediction.

\subsection{FSDAF}

In FSDAF [8], the input data include one pair of coarse and fine resolution images acquired at $t_{1}$ and one coarse-resolution image at $t_{2}$ (Figure 1). The output is a predicted fine-resolution image at $t_{2}$. In this study, the coarse-resolution image is Planet image while the fine-resolution image is WV image. FSDAF includes six main steps: (1) classify the fine-resolution image at $t_{1}$ by an unsupervised classifier, Iterative Self-Organizing Data Analysis Technique Algorithm (ISODATA) [19]; (2) estimate the temporal change of each class in the coarse-resolution image from $t_{1}$ to $t_{2}$; (3) predict the fine-resolution image at $t_{2}$ using the class-level temporal change (refereed as temporal prediction) 
and calculate residuals at each coarse pixel. This temporal prediction would be accurate if no abrupt land cover change occurs; (4) predict the fine-resolution image from the coarse image at $t_{2}$ with a Thin Plate Spline (TPS) interpolator. This TPS prediction should be more accurate than the prediction in the previous step if land cover change occurs; (5) distribute the residuals in step 3 based on TPS prediction to update the temporal prediction; and (6) further smooth the temporal prediction to get the final prediction of the fine-resolution image at $t_{2}$ using the similar strategy of STARFM. The number of classes used in Step 2 is based on the output of ISODATA. In ISODATA, users need to set the minimum and maximum number of classes based on their prior knowledge of the image, and then ISODATA will find the optimal number of classes. In Step 6, FSDAF smooths the temporal prediction using the weighted average of the same-class pixels in a neighborhood. The weight is determined by spatial distance. More details can be found in the paper introducing FSDAF [8].

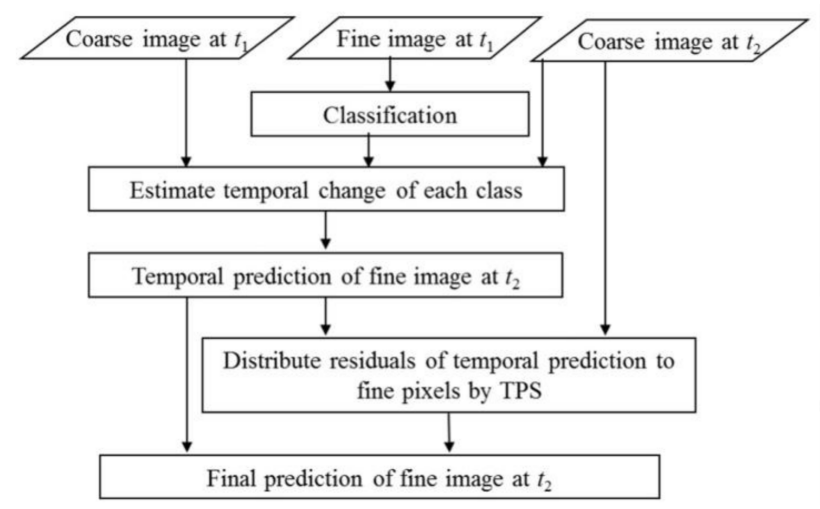

Figure 1. Flowchart of the Flexible Spatiotemporal Data Fusion (FSDAF) fusion algorithm. Figure reproduced from [8].

\subsection{Hybrid Color Mapping (HCM) Approach}

We will use WV and Planet images to illustrate the HCM approach [9]. Figure 2 shows two pairs of Planet (P1 and P2) and WV (W1 and W2) images. The pairs, (P1, W1) and (P2, W2) are collected on the same days. We have two observations. First, the Planet images can be treated as blurred versions of their WV counterparts. Second, the intensity relationship between the Planet pixels is somewhat similar to that of those WV pixels. If we can capture the intensity mapping between Planet images at two different times, then we can use that mapping to predict the WV image at time $t_{p}$ using the WV image at $t_{k}$. It turns out that, although the above idea is simple and intuitive, the prediction results using this idea are quite accurate.

To further justify the above observation, we provide some statistics (means and standard deviations (std)) shown in Tables 1-3 for the Planet and Worldview image pairs. PSE and WVE are Planet and Worldview image pair at an earlier time and PSL and WVL are the image pair at a later time. The numbers are quite close.

Table 1. Means and stds of Red band of two image pairs for the three scenarios in Section 3.

\begin{tabular}{|c|c|c|c|c|c|}
\hline & & Image & CANAL & FIELD & RUNWAY \\
\hline \multirow[t]{8}{*}{ Red Band } & MEAN & PSE & 0.302202 & 0.383428 & 0.269307 \\
\hline & & WVE & 0.303498 & 0.381238 & 0.263837 \\
\hline & & PSL & 0.244982 & 0.356089 & 0.268762 \\
\hline & & WVL & 0.244404 & 0.354388 & 0.243716 \\
\hline & STD & PSE & 0.096934 & 0.084006 & 0.093859 \\
\hline & & WVE & 0.099734 & 0.085258 & 0.093664 \\
\hline & & PSL & 0.082842 & 0.089564 & 0.093986 \\
\hline & & WVL & 0.083615 & 0.089764 & 0.088597 \\
\hline
\end{tabular}




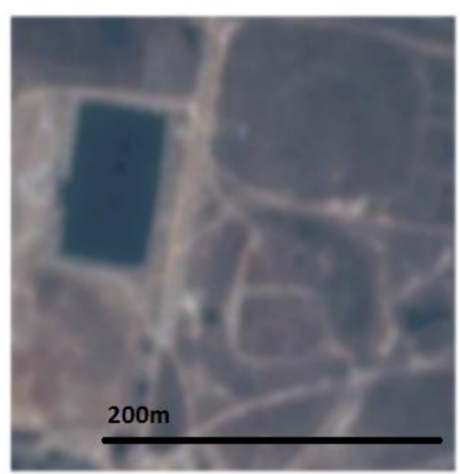

P1

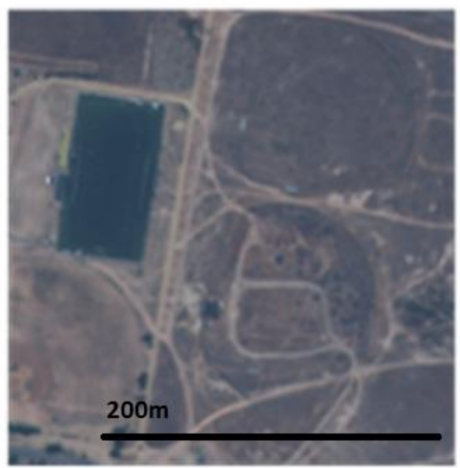

W1

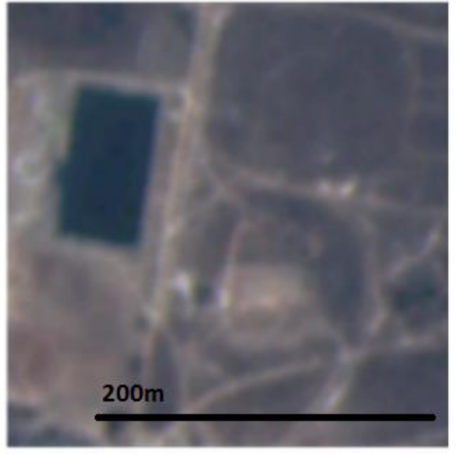

P2

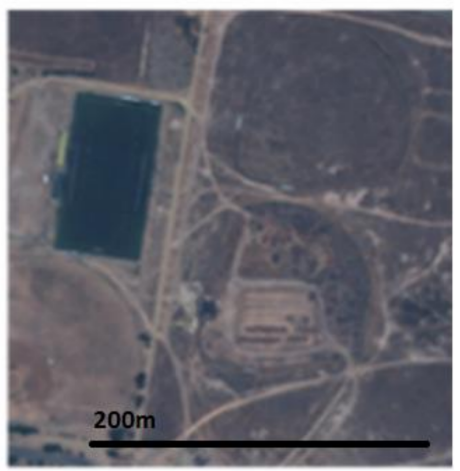

W2

Figure 2. Relationship between two pairs of Planet (top row) and Worldview-2 (WV) (bottom row) images.

Table 2. Means and stds of Green band of two image pairs for the three scenarios in Section 3.

\begin{tabular}{|c|c|c|c|c|c|}
\hline & & Image & CANAL & FIELD & RUNWAY \\
\hline \multirow[t]{8}{*}{ Green Band } & MEAN & PSE & 0.303195 & 0.390444 & 0.273252 \\
\hline & & WVE & 0.304829 & 0.387911 & 0.26864 \\
\hline & & PSL & 0.251461 & 0.354358 & 0.272753 \\
\hline & & WVL & 0.250843 & 0.353061 & 0.250539 \\
\hline & STD & PSE & 0.090393 & 0.064076 & 0.093683 \\
\hline & & WVE & 0.093879 & 0.064833 & 0.09259 \\
\hline & & PSL & 0.075665 & 0.070846 & 0.093826 \\
\hline & & WVL & 0.07582 & 0.069963 & 0.085556 \\
\hline
\end{tabular}

Table 3. Means and stds of Blue band of two image pairs for the three scenarios in Section 3.

\begin{tabular}{|c|c|c|c|c|c|}
\hline & & Image & CANAL & FIELD & RUNWAY \\
\hline \multirow[t]{8}{*}{ Blue Band } & MEAN & PSE & 0.321027 & 0.431879 & 0.295805 \\
\hline & & WVE & 0.322543 & 0.42958 & 0.293019 \\
\hline & & PSL & 0.269751 & 0.392876 & 0.295402 \\
\hline & & WVL & 0.268997 & 0.390579 & 0.275519 \\
\hline & STD & PSE & 0.084322 & 0.049597 & 0.0866 \\
\hline & & WVE & 0.086584 & 0.050008 & 0.084989 \\
\hline & & PSL & 0.069209 & 0.055778 & 0.086771 \\
\hline & & WVL & 0.06806 & 0.055536 & 0.077771 \\
\hline
\end{tabular}

Figure 3 illustrates the HCM approach. Based on the available Planet images collected at $t_{k}$ and $t_{p}$, we learn the pixel by pixel mapping between the two images. The learned matrix, $F$, is then applied 
in the prediction step. Using similar notations in earlier equations, the prediction of the WV image at $t_{p}$ can be achieved by

$$
W\left(x, y, t_{p}\right)=F \times W\left(x, y, t_{k}\right)
$$

where $W(\cdot, \cdot, \cdot)$ denotes a pixel vector (up to $K$ with $K$ being the number of bands) for this application and $F$ is a pixel to pixel mapping/transformation matrix with appropriate dimensions. $F$ can be determined by using the following relationship:

$$
P\left(x, y, t_{p}\right)=F \times P\left(x, y, t_{k}\right)
$$

where $P(\cdot, \cdot, \cdot)$ denotes a pixel vector (K bands). To account for intensity differences between two images, a variant of Equation (4) can be described as

$$
P\left(x, y, t_{p}\right)=F_{1} \times P\left(x, y, t_{k}\right)+F_{2}
$$

where $F_{2}$ is a vector of constants. Procedures to obtain $F$ can be found in [9].

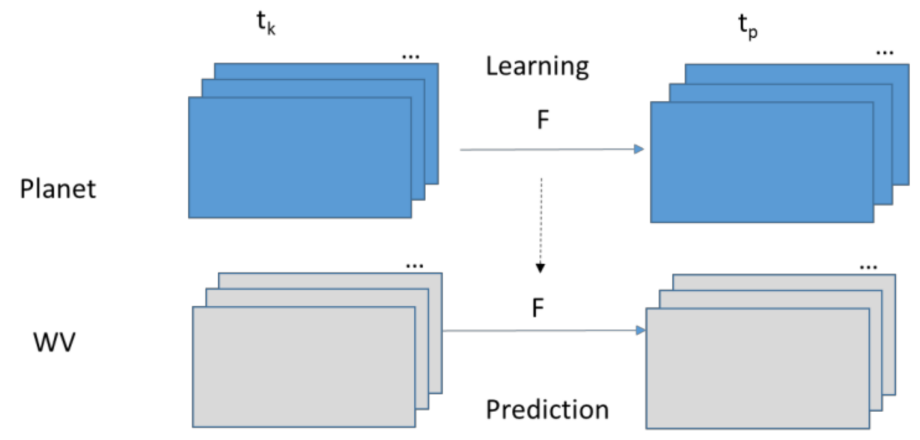

Figure 3. The Hybrid Color Mapping (HCM) approach for image fusion.

Based on our observations, in some cases, prediction results will be more accurate if we divide the images into patches. Each patch will have its own mapping matrix. Figure 4 illustrates the local prediction approach. The patches can be overlapped or non-overlapped. Moreover, for each local patch, which can be a single band or a multi-band image, we use the same estimation algorithm [9] to determine the local mapping matrix, $F_{i}$.

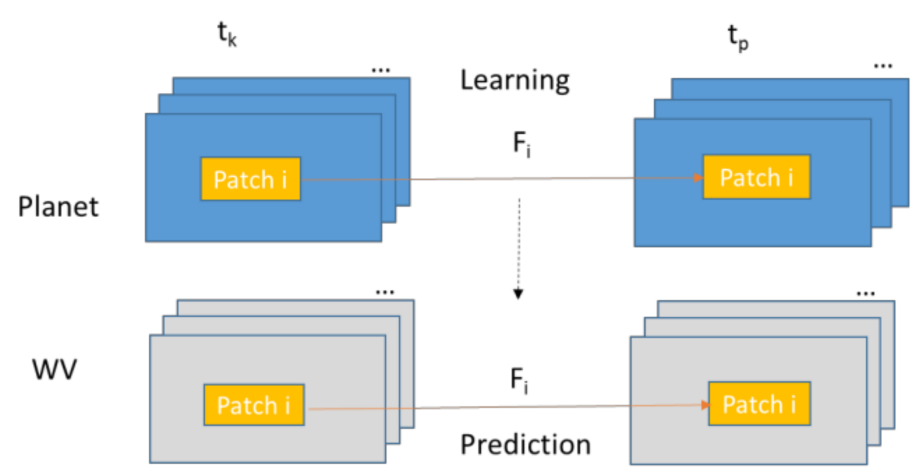

Figure 4. Proposed prediction approach based on local mapping.

\subsection{Performance Metrics}

Although there are many performance metrics in the literature, we selected the following ones: peak signal-to-noise ratio (PSNR) [1], structural similarity (SSIM) [8], Q2N [9], absolution difference (AD) [9], root mean squared error (RMSE) [18], spectral angle mapper (SAM) [18], cross correlation 
(CC) [18], Erreur Relative Globale Adimensionnelle de Synthese (ERGAS) [18], In addition to the above metrics, we also use SSIM maps to visualize the similarity between two images.

- Absolute Difference (AD). The AD of two vectorized images $\mathbf{S}$ (ground truth) and $\hat{\mathbf{S}}$ (prediction) is defined as

$$
\mathrm{AD}(\mathbf{S}, \hat{\mathbf{S}})=\frac{1}{Z} \sum_{j=1}^{Z}\left|s_{j}-\hat{s}_{j}\right|
$$

where $Z$ is the number of pixels in each image. The ideal value of $A D$ is 0 if the prediction is perfect.

- $\quad$ RMSE (Root Mean Squared Error). The RMSE of two vectorized images S (ground truth) and $\hat{\mathbf{S}}$ (prediction) is defined as

$$
\operatorname{RMSE}(\mathbf{S}, \hat{\mathbf{S}})=\sqrt{\frac{1}{Z} \sum_{j=1}^{Z}\left(s_{j}-\hat{s}_{j}\right)^{2}}
$$

where $Z$ is the number of pixels in each image. The ideal value of RMSE is 0 if the prediction is perfect.

- PSNR (Peak Signal to Noise Ratio). PSNR is related to RMSE defined in (8). If the image pixels are expressed in doubles with values between 0 and 1 , then

$$
\mathrm{PSNR}=20 \log (1 / \operatorname{RMSE}(\mathbf{S}, \hat{\mathbf{S}}))
$$

- CC (Cross-Correlation). We used the codes from Open Remote Sensing website (https:// openremotesensing.net/). The ideal value of CC is 1 if the prediction is perfect.

- $\quad$ ERGAS (Erreur Relative. Globale Adimensionnelle de Synthese). The ERGAS is defined as

$$
\operatorname{EGARS}(\mathbf{S}, \hat{\mathbf{S}})=100 d \frac{\operatorname{RMSE}}{\mu}
$$

for some constant $d$ depending on the resolution and $\mu$ is the mean of the ground truth images. The ideal value of ERGAS is 0 if a prediction algorithm is perfect.

- SSIM (Structural Similarity). This is a metric to reflect the similarity between two images. An equation for SSIM can be found in [8]. The ideal value of SSIM is 1 for perfect prediction. We also use the SSIM map to display the similarity values at each pixel location. Bright pixels have high similarity.

- SAM (Spectral Angle Mapper). The spectral angle mapper measures the angle between two vectors. The ideal value of SAM is 0 for perfect reconstruction. For single bands, the angle is zero between two scalar pixels. The exact definition of SAM can be found in [17].

- Q2N: A definition for Q2N can be found in [17]. The ideal value of Q2N is 1. The codes can be downloaded from Open Remote Sensing website (http:/ / openremotesensing.net/).

\section{Results}

In this section, we present some experimental results. Since our main interest is in forward prediction, we only compare with STARFM and FSDAF, which require one pair of Worldview and Planet images at an earlier time, and one Planet image at the time of prediction. We did not compare with the ESTARFM and STAARCH methods, which, in addition to requiring one Planet image at the prediction time, also require two pairs of Worldview and Planet images: one pair at an earlier time and another pair at a future time. In other words, ESTARFM and STAARCH are suitable for processing archived data, not for forward prediction.

In order to make our paper self-contained, we include the following specifications of Planet and Worldview images in Table 4. Only four bands have similar spectral ranges. 
Table 4. Band specifications of Planet and Worldview images.

\begin{tabular}{cccccc}
\hline \multicolumn{2}{c}{$\begin{array}{c}\text { Planet Image } \\
\text { Resolution) }\end{array}$ with $\mathbf{4}$ Bands } & \multicolumn{4}{c}{ Worldview-2 Image (2 m Resolution) with 8 Bands } \\
\hline Blue & $455-515 \mathrm{~nm}$ & Coastal & $400-450 \mathrm{~nm}$ & Red & $630-690 \mathrm{~nm}$ \\
Green & $500-590 \mathrm{~nm}$ & Blue & $450-510 \mathrm{~nm}$ & Red Edge & $705-745 \mathrm{~nm}$ \\
Red & $590-670 \mathrm{~nm}$ & Green & $510-580 \mathrm{~nm}$ & Near-IR1 & $770-895 \mathrm{~nm}$ \\
NIR & $780-860 \mathrm{~nm}$ & Yellow & $585-625 \mathrm{~nm}$ & Near-IR2 & $860-1040 \mathrm{~nm}$ \\
\hline
\end{tabular}

We used WV-2 images that have eight bands, of which we only used the RGB bands for ease of demonstration/visualization. In addition, because Planet images only have four bands, using the RGB bands in both WV-2 and Planet images will be sufficient for both visualization and performance evaluation. The spatial resolution of WV-2 images is $2 \mathrm{~m}$. Most of the Worldview images are off-nadir images in order to have a large coverage area. Moreover, because there is only one Worldview satellite, the revisit times are not as frequent as the Planet satellite constellation of 120 Cubesats.

Each PlanetScope satellite is a CubeSat $3 U$ form factor $(10 \mathrm{~cm}$ by $10 \mathrm{~cm}$ by $30 \mathrm{~cm})$. The complete PlanetScope constellation of approximately 120 satellites will be able to scan the entire land surface of the Earth every day [20]. In Planet image archive, there are Rapideye and Planetscope images. In this study, we used Planetscope images (orthorectified). The image resolution is $3.125 \mathrm{~m}$. For Planet images, only top of atmosphere radiance (TOAR) data products are available. For WV images, surface reflectance images are available. In order to use compatible data, our collaborators at Digital Globe generated WV TOAR images for this study.

Our area of interest is a border area near Tijuana and San Diego. From data archives of both Planet and Digital Globe, the following images were used in our study:

Planet images: 19 July 2017, 27 July 2017, and 5 August 2017;

WV images: 18 July 2017, 26 July 2017, and 3 August 2017

It should be noted that it is difficult to retrieve Planet and WV images for the same dates. However, this also justifies our research, as our goal is to generate high spatial resolution images when high resolution WV images are not available. It is also emphasized that the registration of the two different types of satellite images is non-trivial, as WV images are not taken at nadir. As a result, automated registration algorithms using corner points from buildings may lead to large registration errors at ground level pixels. In this research, we manually selected ground feature points such as road intersections for image alignment.

From the above collected images, we focused on three specific sub-areas representing different scenarios.

\subsection{Scenario 1: Canal Area}

In this case, we used two Planet images collected on 27 July 2017 and 5 August 2017 and two WV images collected on 26 July 2017 and 8 August 2017. The prediction scenario is summarized in Figure 5. Eight performance metrics were used. Due to resolution differences between Planet and WV images, we applied bicubic interpolation to upsample the Planet images to the same size of the WV images. Table 5 summarizes the performance metrics for RGB bands as well as individual bands. First, it can be seen that all of the three fusion methods have significant improvement (all metrics) over the Planet image at the prediction time. The PSNR values have been improved by $0.6 \mathrm{~dB}$ and the SSIM values improved by more than 0.11 . Other metrics all show improvements over those of bicubic. Second, among the three fusion methods, HCM and FSDAF performed slightly better than STARFM. Figure 6 shows visual comparison of the three fused images with the ground truth $(\mathrm{WV})$ and low resolution Planet images at the prediction time. It can be seen that the three algorithms can generate very high quality predictions. In particular, some small objects inside the green circles were correctly predicted 
using the three algorithms. In Figure 7, we also generated SSIM maps for this scenario. Bright indices show high similarity values. One can see that the HCM, FSDAF, and STARFM all have better SSIM values than that of bicubic interpolation.

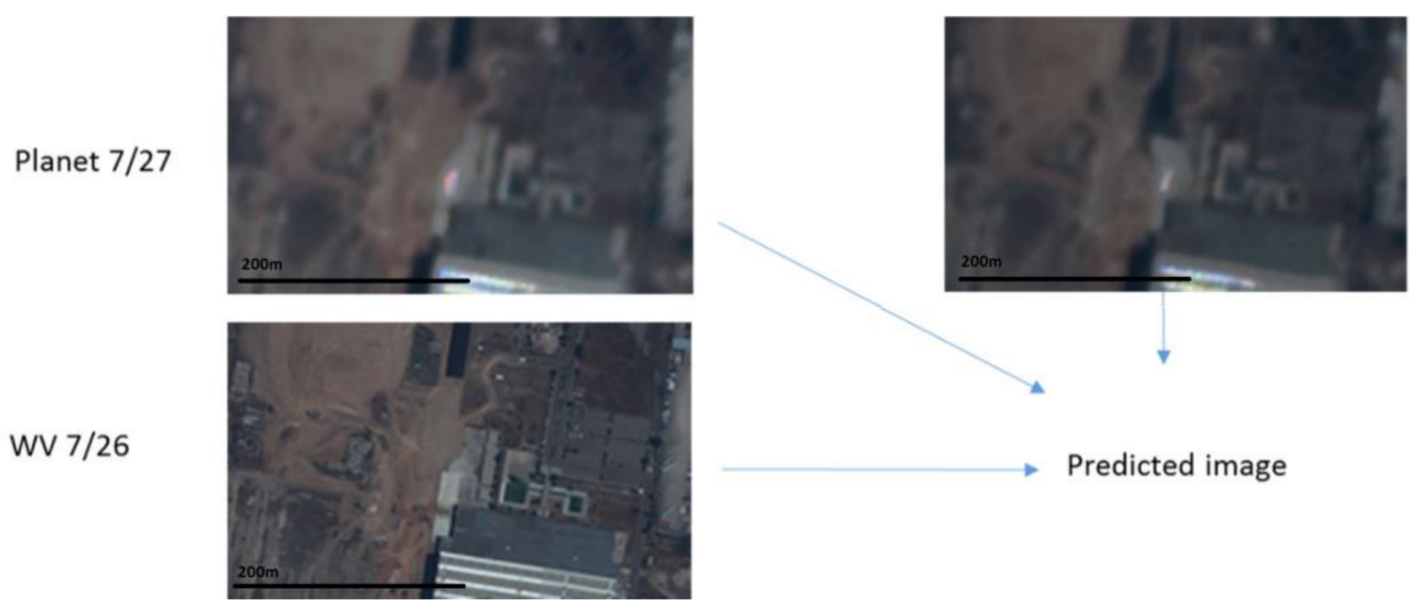

Planet 8/5

Figure 5. Two Planets images at 27 July and 5 August and one WV image at 26 July are fused to generate a prediction. The prediction is then compared to a WV image collected on 3 August 2017.
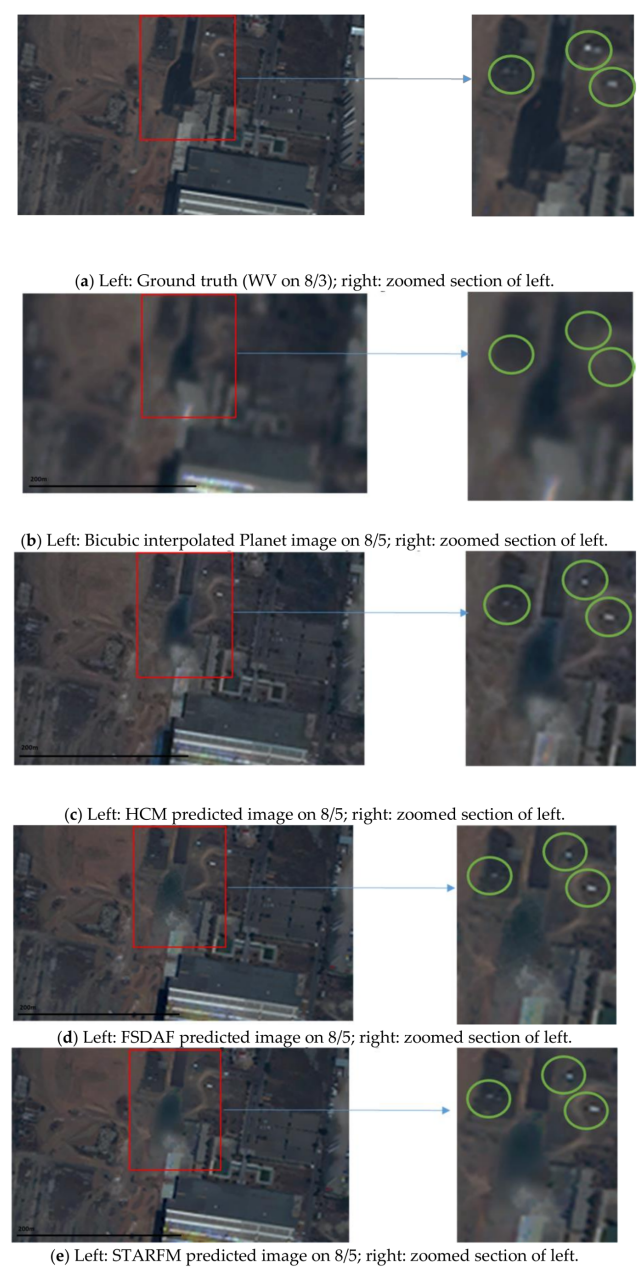

Figure 6. Comparison of different fused images with the ground truth (WV) and the low resolution Planet image at the prediction time. Canal area. 
Table 5. Comparison of prediction results of using Planet and WV images for the scenario depicted in Figure 5. Bold characters indicate the best performing method for that row.

\begin{tabular}{cccccccccc}
\hline \multicolumn{3}{c}{ RGB } & \multicolumn{3}{c}{$\mathbf{R}$} \\
\hline \multicolumn{3}{c}{ Canal } & & & \multicolumn{5}{c}{ Canal } \\
RMSE & STARFM & FSDAF & HCM & Bicubic & & STARFM & FSDAF & HCM & Bicubic \\
PSNR & 28.4721 & 28.5595 & $\mathbf{2 8 . 5 7 6 0}$ & 27.9017 & PSNR & 27.9065 & 27.8942 & $\mathbf{2 8 . 2 1 3 2}$ & 27.0623 \\
AD & 0.0221 & 0.0222 & $\mathbf{0 . 0 2 2 1}$ & 0.0259 & AD & $\mathbf{0 . 0 2 4 1}$ & 0.0245 & 0.0242 & 0.0303 \\
CC & 0.4384 & $\mathbf{0 . 4 4 1 1}$ & 0.4368 & 0.4229 & CC & 0.8499 & 0.8487 & $\mathbf{0 . 8 5 9 9}$ & 0.8199 \\
SAM & 2.1033 & $\mathbf{2 . 0 2 7 4}$ & 2.3677 & 2.3576 & SSIM & $\mathbf{0 . 8 2 3 0}$ & 0.8200 & 0.8138 & 0.6687 \\
SSIM & 0.8491 & $\mathbf{0 . 8 4 9 3}$ & 0.8350 & 0.7366 & ERGAS & 16.7615 & $\mathbf{1 6 . 9 2 7 2}$ & 16.2325 & 18.5180 \\
ERGAS & 15.2969 & 15.2785 & $\mathbf{1 5 . 0 9 1 9}$ & 16.4045 & Q2N & 0.7844 & $\mathbf{0 . 7 8 0 6}$ & 0.7906 & 0.6755 \\
Q2N & 0.7795 & $\mathbf{0 . 7 8 1 4}$ & 0.7797 & 0.6737 & & & & &
\end{tabular}

(a) Metrics for all 3 bands

(b) Metrics for Red band

\begin{tabular}{cccccccccc}
\hline \multicolumn{3}{c}{ G } & \multicolumn{3}{c}{ B } \\
\hline \multicolumn{3}{c}{ Canal } & & & & \multicolumn{5}{c}{ Canal } \\
RMSE & STARFM & FSDAF & HCM & Bicubic & & STARFM & FSDAF & HCM & Bicubic \\
PSNR & 28.5357 & 28.5890 & $\mathbf{2 8 . 8 0 8 3}$ & 28.0401 & PSNR & 29.0499 & $\mathbf{2 9 . 3 1 0 2}$ & 28.7308 & 28.7733 \\
AD & 0.0220 & 0.0222 & $\mathbf{0 . 0 2 0 8}$ & 0.0255 & AD & 0.0204 & $\mathbf{0 . 0 2 0 1}$ & 0.0214 & 0.0220 \\
CC & 0.8089 & 0.8104 & $\mathbf{0 . 8 2 0 6}$ & 0.7836 & CC & 0.7692 & $\mathbf{0 . 7 7 6 6}$ & 0.7567 & 0.7403 \\
SSIM & $\mathbf{0 . 8 4 4 3}$ & 0.8429 & 0.8380 & 0.7148 & SSIM & $\mathbf{0 . 8 4 4 8}$ & 0.8443 & 0.8178 & 0.7413 \\
ERGAS & 15.3994 & 15.4212 & $\mathbf{1 4 . 9 5 2 7}$ & 16.3709 & ERGAS & 13.5607 & $\mathbf{1 3 . 2 6 4 8}$ & 14.0079 & 14.0142 \\
Q2N & 0.7592 & 0.7546 & $\mathbf{0 . 7 8 1 1}$ & 0.6686 & Q2N & 0.7237 & 0.7177 & $\mathbf{0 . 7 2 8 4}$ & 0.6468 \\
\multicolumn{3}{c}{ (c) Metrics for Green band } & & \multicolumn{3}{c}{ (d) Metrics for Blue band } & \\
\hline
\end{tabular}

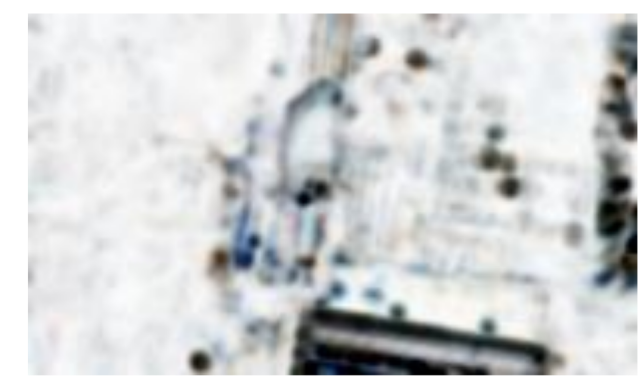

(a) STARFM

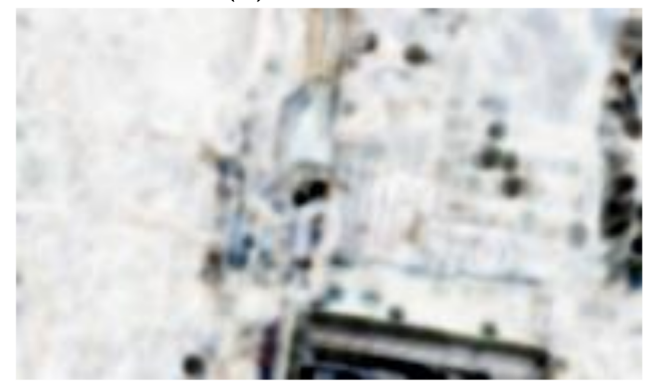

(c) $\mathrm{HCM}$

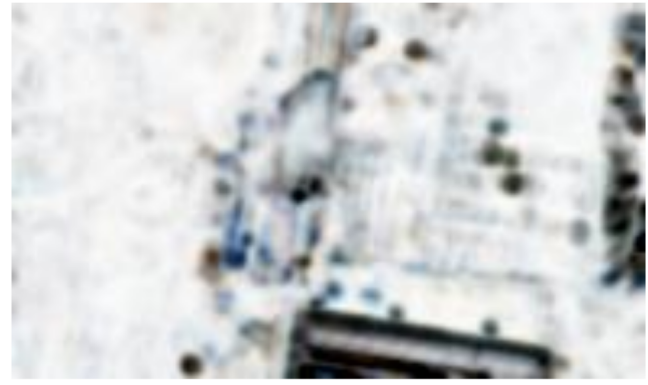

(b) FSDAF

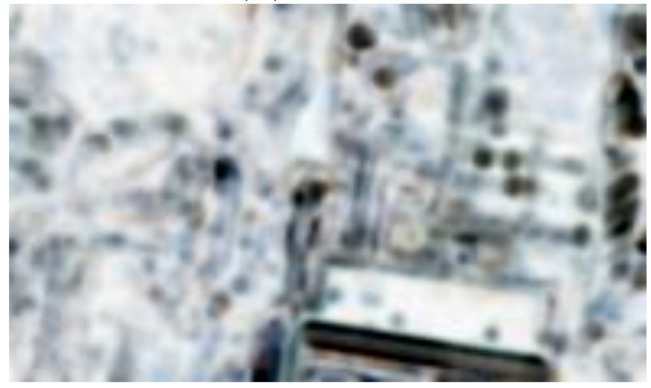

(d) Bicubic

Figure 7. SSIM (Structural Similarity) maps of the Canal scenario using different methods. (a-d) are the maps for STARFM, FSDAF, HCM, and bicubic, respectively.

\subsection{Scenario 2: Field Area}

In this scenario, we used two Planet images collected at 19 July and 27 July and one WV image at 18 July to jointly predict a high resolution image at $27 \mathrm{July}$. Because there is no high resolution WV image at 27 July, we used the WV image at 26 July as the ground truth for generating the performance 
metrics. The prediction scenario is illustrated in Figure 8. From Table 6, one can see that the three fusion algorithms have improved the PSNRs by about $3 \mathrm{dBs}$ over the bicubic interpolated Planet image. The SSIM values have also been improved by close to $50 \%$. Other metrics have all been improved over the bicubic outputs. By closely inspecting the various images in Figure 9, the fused images can recover more fine details, which were blurred and hidden in the original Planet image at the prediction time. Figure 10 shows the SSIM maps of different methods. We can see that the improvement over bicubic is quite significant.

Planet $7 / 19$
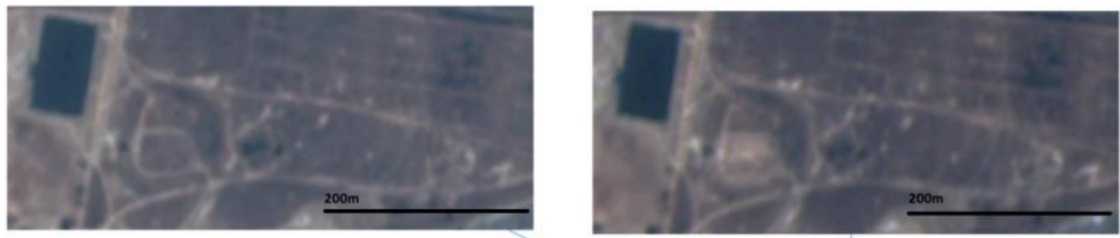

Planet $7 / 27$

WV $7 / 18$
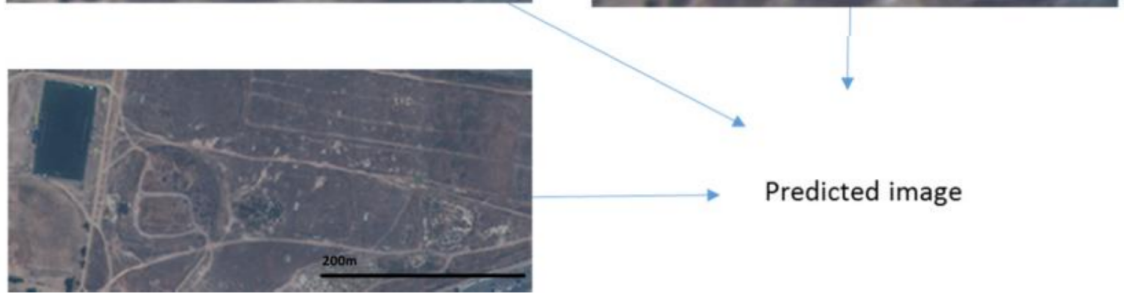

Predicted image

Figure 8. Two Planets images at 19 July and 27 July and one WV image at 18 July are fused to generate a prediction. The prediction is then compared to a WV image collected on 26 July 2017.

Table 6. Comparison of prediction results of using Planet and WV images for the scenario depicted in Figure 8.

\begin{tabular}{|c|c|c|c|c|c|c|c|c|c|}
\hline \multicolumn{5}{|c|}{ RGB } & \multicolumn{5}{|c|}{$\mathbf{R}$} \\
\hline \multicolumn{5}{|c|}{ Field } & \multicolumn{5}{|c|}{ Field } \\
\hline & STARFM & FSDAF & $\mathrm{HCM}$ & Bicubic & & STARFM & FSDAF & $\mathrm{HCM}$ & Bicubic \\
\hline RMSE & 0.0302 & 0.0286 & 0.0266 & 0.0479 & RMSE & 0.0337 & 0.0321 & 0.0308 & 0.0560 \\
\hline PSNR & 30.4069 & 30.8705 & 31.5069 & 26.3870 & PSNR & 29.4494 & 29.8801 & 30.2383 & 25.0355 \\
\hline $\mathrm{AD}$ & 0.0206 & 0.0194 & 0.0168 & 0.0335 & $\mathrm{AD}$ & 0.0233 & 0.0220 & 0.0196 & 0.0400 \\
\hline CC & 0.4967 & 0.5025 & 0.5121 & 0.4223 & CC & 0.9300 & 0.9360 & 0.9394 & 0.8047 \\
\hline SAM & 1.4415 & 1.2646 & 1.0583 & 1.7460 & SSIM & 0.8661 & 0.8723 & 0.8926 & 0.5412 \\
\hline SSIM & 0.8902 & 0.8966 & 0.9156 & 0.6574 & ERGAS & 9.5117 & 9.0515 & 8.7089 & 15.8107 \\
\hline ERGAS & 8.3115 & 7.8876 & 7.3729 & 13.2696 & Q2N & 0.8602 & 0.8712 & 0.8967 & 0.5391 \\
\hline \multirow[t]{2}{*}{ Q2N } & 0.8439 & 0.8559 & 0.8929 & 0.5461 & & & & & \\
\hline & \multicolumn{4}{|c|}{ (a) Metrics for all 3 bands } & \multicolumn{5}{|c|}{ (b) Metrics for Red band } \\
\hline \multicolumn{5}{|c|}{ G } & \multicolumn{5}{|c|}{ B } \\
\hline \multicolumn{5}{|c|}{ Field } & \multicolumn{5}{|c|}{ Field } \\
\hline & STARFM & FSDAF & $\mathrm{HCM}$ & Bicubic & & STARFM & FSDAF & $\mathrm{HCM}$ & Bicubic \\
\hline RMSE & 0.0286 & 0.0274 & 0.0259 & 0.0464 & RMSE & 0.0279 & 0.0260 & 0.0225 & 0.0400 \\
\hline PSNR & 30.8846 & 31.2412 & 31.7407 & 26.6616 & PSNR & 31.0748 & 31.7023 & 32.9749 & 27.9594 \\
\hline $\mathrm{AD}$ & 0.0195 & 0.0187 & 0.0166 & 0.0328 & $\mathrm{AD}$ & 0.0189 & 0.0176 & 0.0141 & 0.0276 \\
\hline CC & 0.9180 & 0.9240 & 0.9292 & 0.7820 & $\mathrm{CC}$ & 0.8760 & 0.8895 & 0.9150 & 0.7418 \\
\hline SSIM & 0.8827 & 0.8868 & 0.9047 & 0.6042 & SSIM & 0.8619 & 0.8713 & 0.9065 & 0.6407 \\
\hline ERGAS & 8.0951 & 7.7694 & 7.3551 & 13.1633 & ERGAS & 7.1583 & 6.6595 & 5.7564 & 10.2467 \\
\hline \multirow[t]{2}{*}{ Q2N } & 0.8522 & 0.8607 & 0.8885 & 0.5361 & Q2N & 0.8054 & 0.8209 & 0.8782 & 0.5155 \\
\hline & \multicolumn{4}{|c|}{ (c) Metrics for Green band } & \multicolumn{5}{|c|}{ (d) Metrics for Blue band } \\
\hline
\end{tabular}




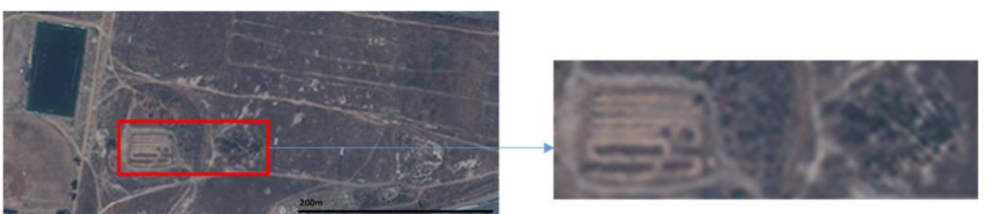

(a) Left: Ground truth (WV on 7/26); right: zoomed section of left.

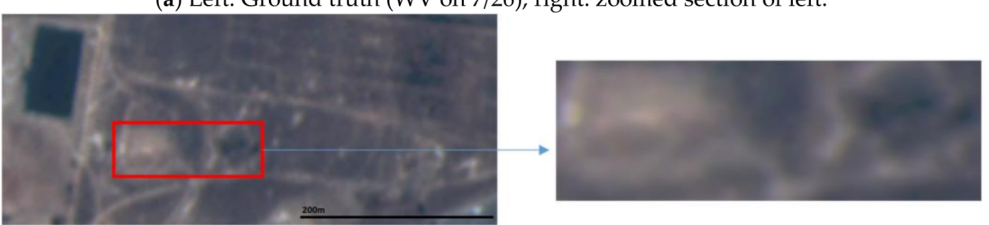

(b) Left: Bicubic interpolated Planet image on 7/27; right: zoomed section of left.

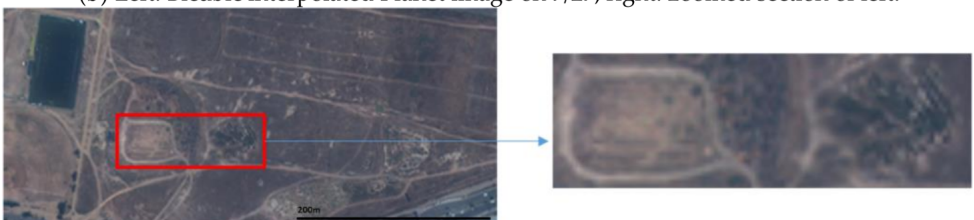

(c) Left: HCM predicted image on 7/27; right: zoomed section of left.

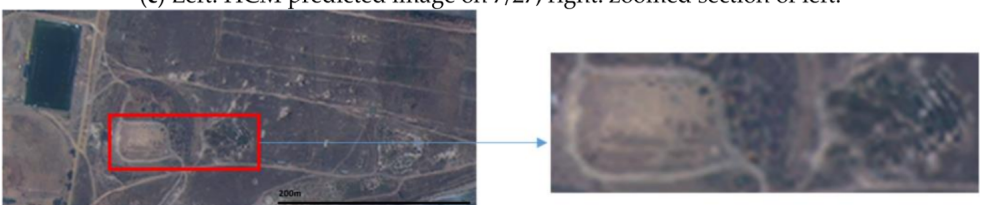

(d) Left: FSDAF predicted image on 7/27; right: zoomed section of left.

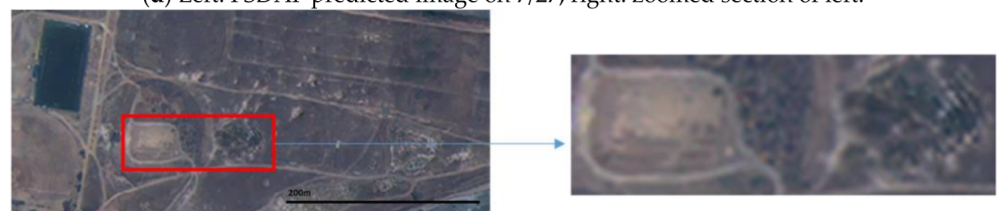

(e) Left: STARFM predicted image on 7/27; right: zoomed section of left.

Figure 9. Comparison of different fused images with the ground truth (WV) and the low resolution Planet image at the prediction time. Field area.

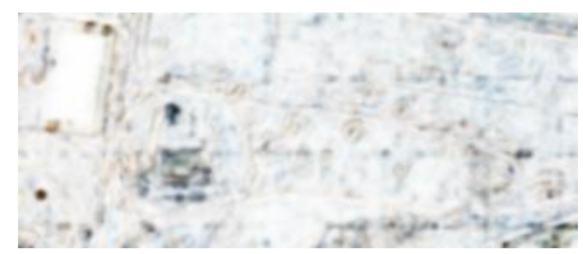

(a) STARFM

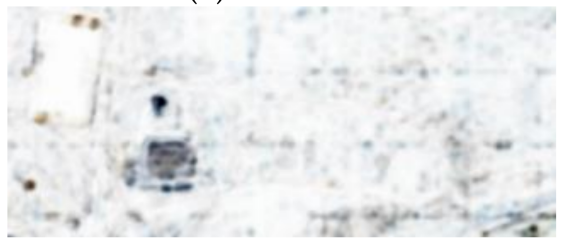

(c) $\mathrm{HCM}$

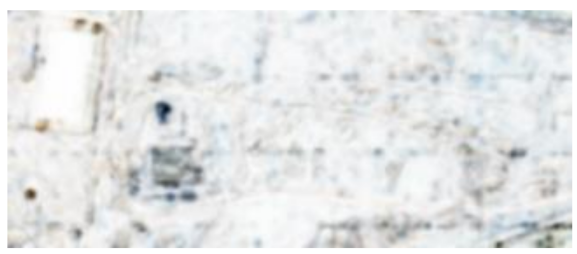

(b) FSDAF

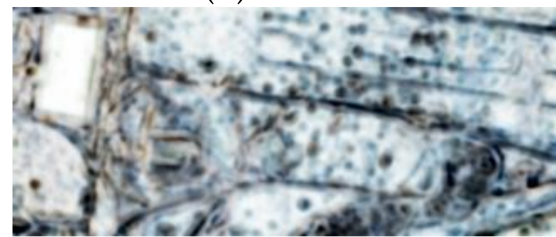

(d) Bicubic

Figure 10. SSIM maps of the Field scenario using different methods. (a-d) are the maps for STARFM, FSDAF, HCM, and bicubic, respectively.

\subsection{Scenario 3: Runway Area}

Here, we used two Planet images at 27 July and 5 August and one WV image at 26 July to generate a prediction at 5 August. Due to lack of a WV image at 5 August, we used the WV image at 3 August as the ground truth. Figure 11 illustrates the prediction scenario. The prediction performance metrics 
are summarized in Table 7. In terms of PSNR, all three fusion algorithms improved over the Planet image by $3 \mathrm{dBs}$. In terms of SSIM, the improvement is close to $10 \%$. Other metrics show a similar trend as the above metrics. In terms of subjective evaluation, one can see from Figure 12 that the fusion algorithms can recover the fine details whereas the Planet image missed quite a few of the detailed features inside the red bounding box.

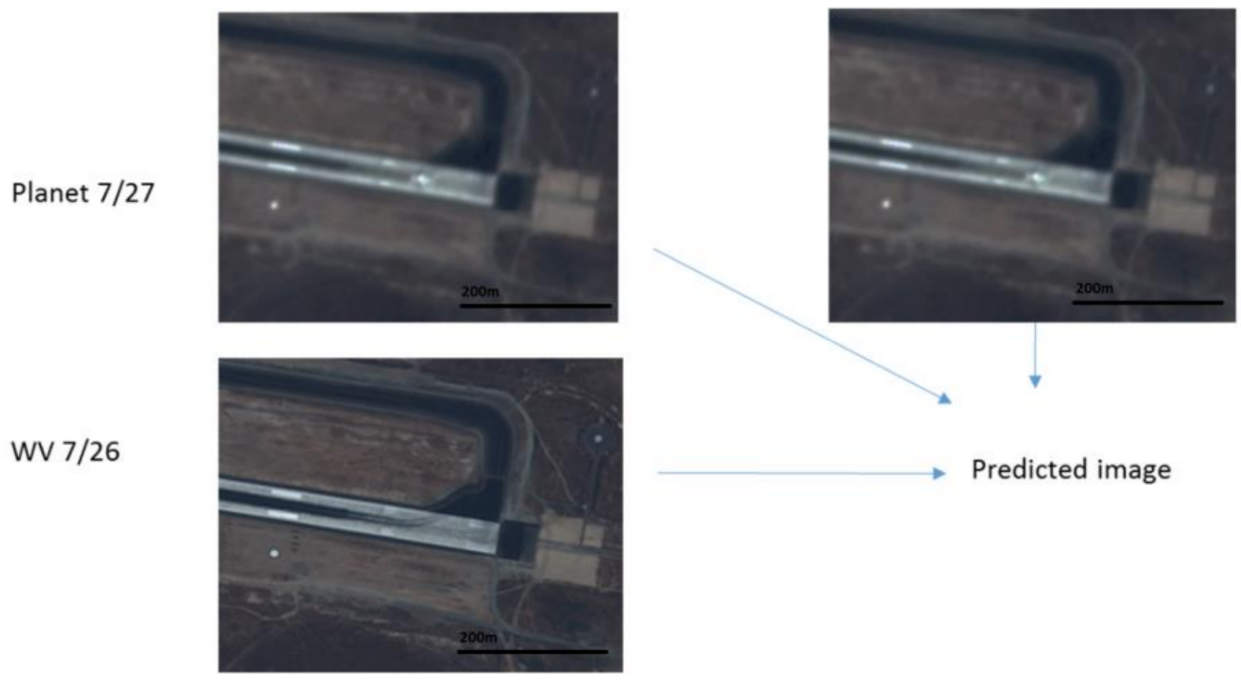

Planet $8 / 5$

Figure 11. Two Planets images at 27 July and 5 August and one WV image at 26 July are fused to generate a prediction. The prediction is then compared to a WV image collected on 3 August 2017.

Table 7. Comparison of prediction results of using Planet and WV images for the scenario depicted in Figure 11.

\begin{tabular}{|c|c|c|c|c|c|c|c|c|c|}
\hline \multicolumn{5}{|c|}{ RGB } & \multicolumn{5}{|c|}{$\mathbf{R}$} \\
\hline \multicolumn{5}{|c|}{ Runway } & \multicolumn{5}{|c|}{ Runway } \\
\hline & STARFM & FSDAF & $\mathrm{HCM}$ & Bicubic & & STARFM & FSDAF & $\mathrm{HCM}$ & Bicubic \\
\hline RMSE & 0.0327 & 0.0316 & 0.0329 & 0.0457 & RMSE & 0.0344 & 0.0333 & 0.0348 & 0.0489 \\
\hline PSNR & 29.7128 & 30.0081 & 29.6460 & 26.8029 & PSNR & 29.2637 & 29.5449 & 29.1655 & 26.2078 \\
\hline $\mathrm{AD}$ & 0.0231 & 0.0218 & 0.0231 & 0.0304 & $\mathrm{AD}$ & 0.0255 & 0.0242 & 0.0256 & 0.0336 \\
\hline $\mathrm{CC}$ & 0.5300 & 0.5301 & 0.5293 & 0.5031 & CC & 0.9534 & 0.9534 & 0.9517 & 0.8967 \\
\hline SAM & 1.3649 & 1.3469 & 1.3208 & 1.8684 & SSIM & 0.8451 & 0.8451 & 0.8417 & 0.7209 \\
\hline SSIM & 0.8896 & 0.8892 & 0.8862 & 0.7903 & ERGAS & 14.0951 & 13.6461 & 13.2019 & 20.0384 \\
\hline ERGAS & 12.8138 & 12.3881 & 12.0533 & 17.9663 & Q2N & 0.6589 & 0.6783 & 0.7852 & 0.5326 \\
\hline Q2N & 0.7932 & 0.8020 & 0.8081 & 0.6833 & & & & & \\
\hline \multicolumn{5}{|c|}{ (a) Metrics for all 3 bands } & \multicolumn{5}{|c|}{ (b) Metrics for Red band } \\
\hline \multicolumn{5}{|c|}{ G } & \multicolumn{5}{|c|}{ B } \\
\hline \multicolumn{5}{|c|}{ Runway } & \multicolumn{5}{|c|}{ Runway } \\
\hline & STARFM & FSDAF & $\mathrm{HCM}$ & Bicubic & & STARFM & FSDAF & $\mathrm{HCM}$ & Bicubic \\
\hline RMSE & 0.0323 & 0.0313 & 0.0325 & 0.0463 & RMSE & 0.0312 & 0.0301 & 0.0315 & 0.0415 \\
\hline PSNR & 29.8063 & 30.0904 & 29.7745 & 26.6800 & PSNR & 30.1111 & 30.4357 & 30.0453 & 27.6414 \\
\hline $\mathrm{AD}$ & 0.0227 & 0.0214 & 0.0226 & 0.0304 & $\mathrm{AD}$ & 0.0211 & 0.0197 & 0.0211 & 0.0271 \\
\hline $\mathrm{CC}$ & 0.9566 & 0.9568 & 0.9557 & 0.9023 & $\mathrm{CC}$ & 0.9528 & 0.9531 & 0.9513 & 0.9088 \\
\hline SSIM & 0.8758 & 0.8757 & 0.8737 & 0.7656 & SSIM & 0.8908 & 0.8894 & 0.8856 & 0.8023 \\
\hline ERGAS & 12.8810 & 12.4665 & 12.0869 & 18.4612 & ERGAS & 11.3131 & 10.8981 & 10.7453 & 15.0338 \\
\hline Q2N & 0.6759 & 0.7228 & 0.8018 & 0.5914 & Q2N & 0.6578 & 0.6973 & 0.7830 & 0.5854 \\
\hline \multicolumn{5}{|c|}{ (c) Metrics for Green band } & \multicolumn{5}{|c|}{ (d) Metrics for Blue band } \\
\hline
\end{tabular}



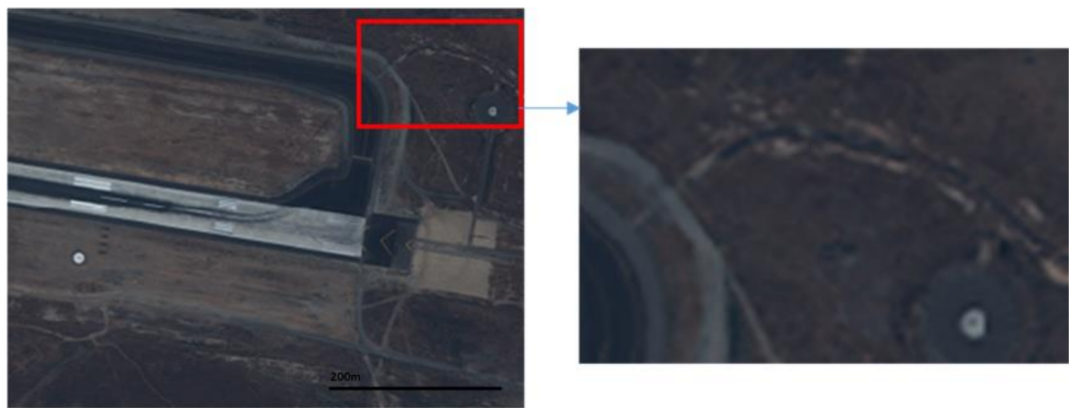

(a) Left: Ground truth (WV on 8/3); right: zoomed section of left.
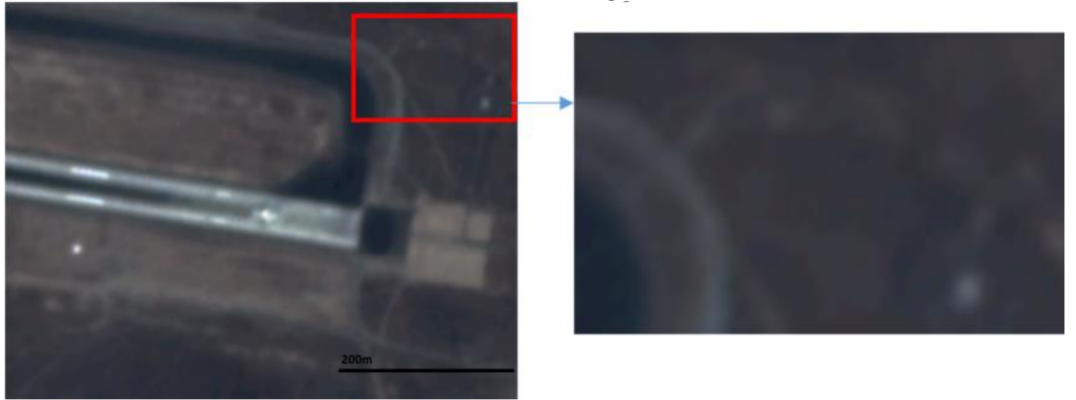

(b) Left: Planet image on 8/5; right: zoomed section of left.
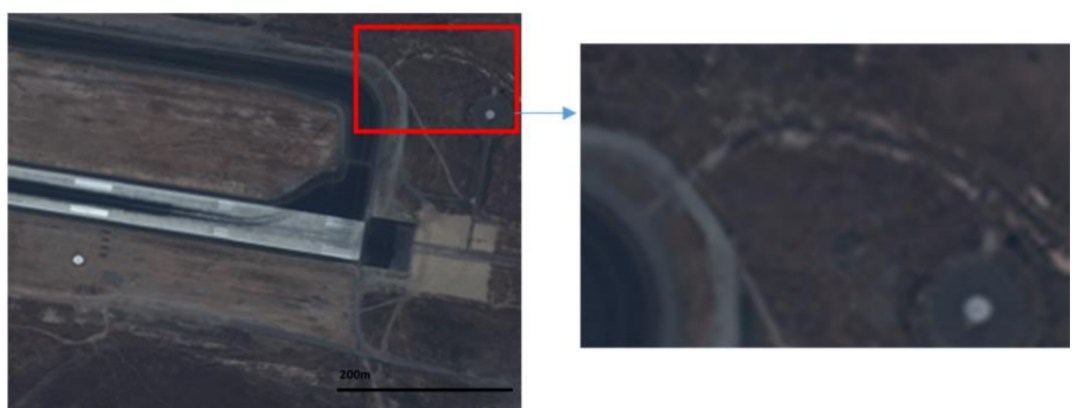

(c) Left: HCM predicted image on 8/5; right: zoomed section of left.
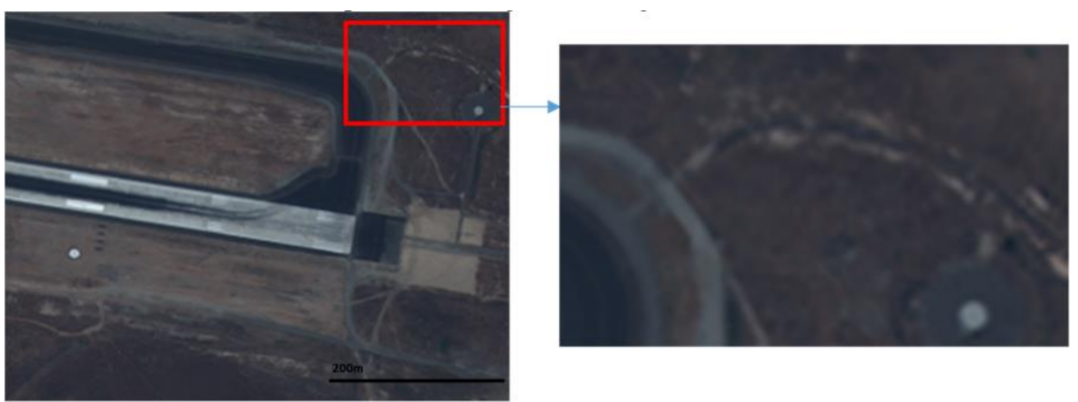

(d) Left: FSDAF predicted image on 8/5; right: zoomed section of left.
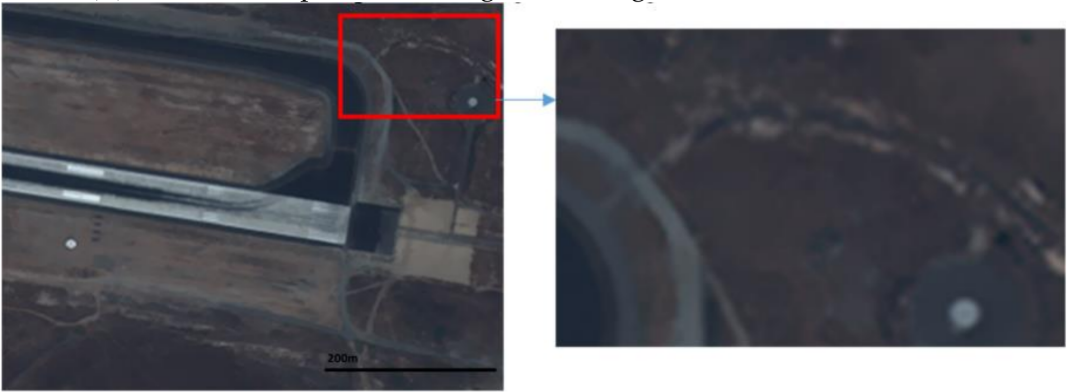

(e) Left: STARFM predicted image on 8/5; right: zoomed section of left.

Figure 12. Comparison of different fused images with the ground truth (WV) and the low resolution Planet image at the prediction time. Runway area. 
Figure 13 shows the SSIM maps, which further corroborate that the HCM, FSDAF, and STARFM all have better performance than bicubic.

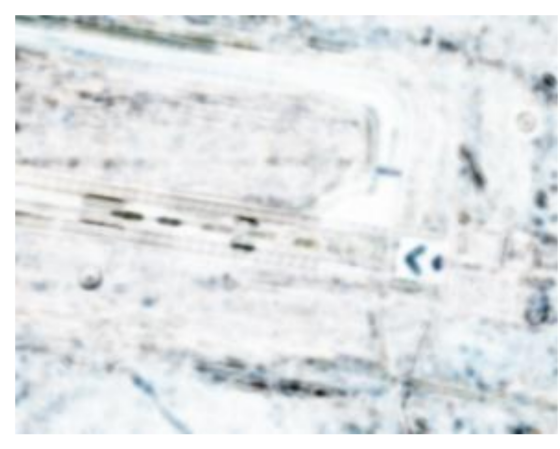

(a) STARFM

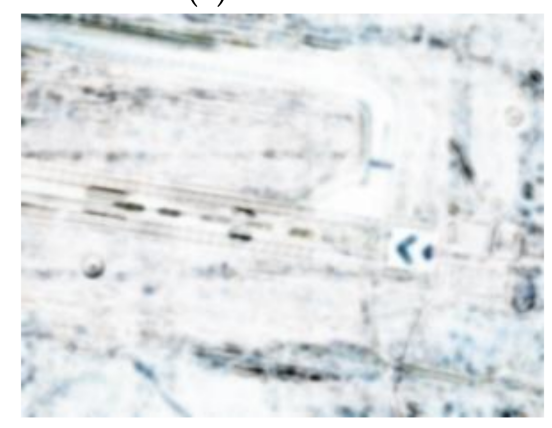

(c) $\mathrm{HCM}$

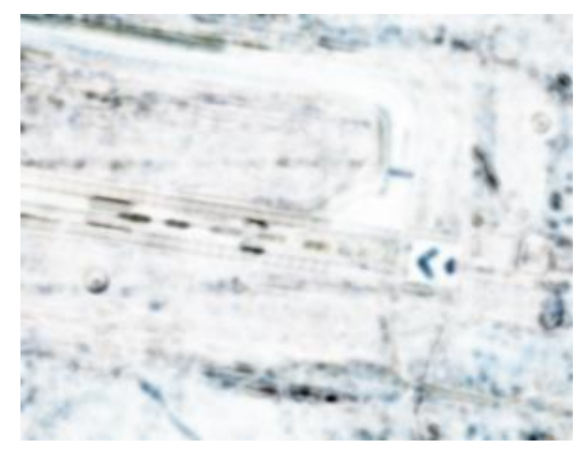

(b) FSDAF

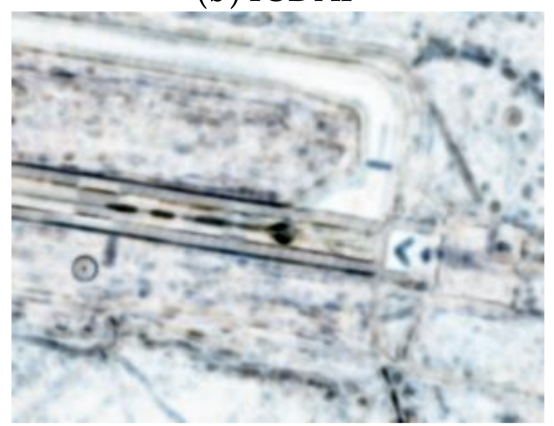

(d) Bicubic

Figure 13. SSIM maps of the Runway scenario using different methods. (a-d) are the maps for STARFM, FSDAF, HCM, and bicubic, respectively.

\section{Discussion}

The study in this paper is important because Planet images are collected using CubeSat's and have more frequent revisit times, as compared to the WV images. However, the resolution of Planet is worse than that of WV images. The methods described in this paper can generate a high spatial resolution and high temporal resolution image series by fusing the two satellite images and will have many important applications, including border monitoring, damage assessment, etc. Decision makers can perform accurate situation assessment based on the fused data.

Although there are quite a few fusion studies on MODIS and Landsat images, no one has carried out a fusion study for Planet and WV images to the best of our knowledge. We presented three approaches to fusing the Planet and WV images. The approaches are representative algorithms in the literature. STARFM is well-known in the fusion of MODIS and Landsat. FSDAF incorporates clustering information to assist the prediction process, but requires more computations. HCM is a relatively simple and efficient algorithm for prediction. Based on the experimental results on three scenarios near a US-Mexico border area, the improvement of the three fusion approaches over the original Planet images is significant. In particular, the PSNR gains are close to $3 \mathrm{~dB}$ (Tables 5-7) for some of the three scenarios. Other performance metrics such as AD, RMSE, SAM, SSIM, Q2N, ERGAS, and CC all show significant improvement over the baseline (bicubic). In addition to the above metrics, we also generated SSIM index maps (Figures 7, 10 and 13), which also show significant visual improvement of the results from HCM, FSDAF, and STARFM over that of the bicubic method. We can also visually see that the prediction images from all of the algorithms can reveal some fine textures that are missing in the Planet images. Moreover, as can be seen from Figures 6, 9 and 12, the fused images have much better visual quality than the original Planet images. 
It is also worth to mention that, from the results in Section 3, one can observe that none of the three methods can perform well under all scenarios. It is therefore important to have a library of algorithms for image fusion. The best algorithm should be selected for a given application.

Since the Planet images are top of atmosphere radiance (TOAR), we opted to work in radiance domain by also using WV-2 images (TOAR). The two types of images indeed have magnitude differences in the radiance domain. To overcome this, we carried out some preprocessing. In pansharpening papers, a common practice has been widely used, which is to apply histogram matching $[16,17]$ between pan band and the multispectral bands. Here, we adopted a similar strategy in order to work directly in radiance domain. The idea is actually very simple. We used W1 as the reference and applied histogram matching to P1 and P2 so that P1 and P2 are matched to W1. Since W1 and W2 are collected at the same time of the day with the same altitude (the Worldview satellite flies over the same area at roughly the same time of the day), they have roughly the same radiance. We then learn the mapping between the adjusted P1 and P2. Finally, we perform the mapping from W1 to W2 by using the learned mapping earlier.

It should be noted that, even if we work directly in the reflectance domain, there is no guarantee that the reflectance values between the Planet and Worldview images will be the same. We may still need to perform some histogram matching after atmospheric compensation. This is because the atmospheric compensation procedures in generating the Worldview reflectance images are proprietary. Digital Globe has a proprietary software known as GBDX (https://gbdxdocs.digitalglobe.com/docs/ advanced-image-preprocessor), which contains atmospheric compensation algorithms. If we use FLAASH in ENVI to generate reflectance images for Planet, the two sets of reflectance images resulting from different atmospheric compensation algorithms may still be slightly different and hence histogram matching may still be required after atmospheric compensation.

Because Worldview images are not collected at nadir for most of the images, the registration and alignment with Planet images should be done carefully. In this research, we performed the alignment manually. One future direction is to develop an automated alignment program that will significantly reduce manual labor.

The image compatibility issue between different satellite images together with the image alignment issue require more in depth studies in the future. The image alignment is non-trivial. Here, our goal is on image fusion algorithm assessment where we assume that the registration is done and the images (regardless whether they are radiance or reflectance) have similar histograms.

Another direction is to apply the high temporal high spatial resolution images to some applications such as damage assessment due to flooding, fire, hurricane, tsunami, etc.

\section{Conclusions}

In this paper, we applied three spatiotemporal data fusion approaches for forward predicting images with high spatial and high temporal resolutions from Planet and WV images. The performance of the three approaches is comparable using actual WV and Planet images. Compared to other fusion approaches such as STAARCH and ESTARFM, the methods tested in this study do not require two pairs of Planet and WV images and are more appropriate for forward prediction.

Acknowledgments: This work was supported in part by DARPA under contract D17PC00025. This paper was approved with Distribution Statement "A" (Approved for Public Release, Distribution Unlimited). The views, opinions and/or findings expressed are those of the author(s) and should not be interpreted as representing the official views or policies of the Department of Defense or the U.S. Government. We would like to thank Planet for providing the satellite images via an educational program with Old Dominion University.

Author Contributions: C.K. conceived the overall approach and wrote the paper. X.Z. provided inputs related to the FSDAF algorithm. F.G. provided the STARFM inputs. B.C. generated all the figures and tables. D.P., J.L., and Y.S. retrieved the relevant Planet images and carried out some preprocessing to those images. K.K. and G.M. provided the Worldview images and pre-processed those images to be compatible with the Planet images.

Conflicts of Interest: The authors declare no conflict of interest. 


\section{References}

1. Kwan, C.; Dao, M.; Chou, B.; Kwan, L.M.; Ayhan, B. Mastcam image enhancement using estimated point spread functions. In Proceedings of the IEEE Ubiquitous Computing, Electronics \& Mobile Communication Conference, New York, NY, USA, 19-21 October 2017.

2. Schowengerdt, R.A. Remote Sensing: Models and Methods for Image Processing; Academic Press: Cambridge, MA, USA, 1997.

3. Gao, F.; Masek, J.; Schwaller, M.; Hall, F. On the blending of the Landsat and MODIS surface reflectance: Predicting daily Landsat surface reflectance. IEEE Trans. Geosci. Remote Sens. 2006, 44, 2207-2218.

4. Hilker, T.; Wulder, A.M.; Coops, N.C.; Linke, J.; McDermid, G.; Masek, J.G.; Gao, F.; White, J.C. A new data fusion model for high spatial- and temporal-resolution mapping of forest disturbance based on Landsat and MODIS. Remote Sens. Environ. 2009, 113, 1613-1627. [CrossRef]

5. Zhu, X.; Chen, J.; Gao, F.; Chen, X.; Masek, J.G. An enhanced spatial and temporal adaptive reflectance fusion model for complex heterogeneous regions. Remote Sens. Environ. 2010, 114, 2610-2623. [CrossRef]

6. Zhang, F.; Zhu, X.; Liu, D. Blending MODIS and Landsat images for urban flood mapping. Int. J. Remote Sens. 2014, 35, 3237-3253. [CrossRef]

7. Gao, F.; Hilker, T.; Zhu, X.; Anderson, M.; Masek, J.; Wang, P.; Yang, Y. Fusing Landsat and MODIS data for vegetation monitoring. IEEE Geosci. Remote Sens. Mag. 2015, 3, 47-60. [CrossRef]

8. Zhu, X.; Helmer, E.; Gao, F.; Liu, D.; Chen, J.; Lefsky, M. A flexible spatiotemporal method for fusing satellite images with different resolutions. Remote Sens. Environ. 2016, 172, 165-177. [CrossRef]

9. Kwan, C.; Budavari, B.; Gao, F.; Zhu, X. A hybrid color mapping approach to fusing MODIS and landsat images for forward prediction. Remote Sens. 2018, 10, 520. [CrossRef]

10. Hazaymeh, K.; Hassan, Q.K. Spatiotemporal image-fusion model for enhancing temporal resolution of Landsat-8 surface reflectance images using MODIS images. J. Appl. Remote Sens. 2015, 9, 096095. [CrossRef]

11. Addesso, P.; Conte, R.; Longo, M.; Restaino, R.; Vivone, G. Sequential Bayesian methods for resolution enhancement of TIR image sequences. IEEE J. Sel. Top. Appl. Earth Obs. Remote Sens. 2015, 8, 233-243. [CrossRef]

12. Wu, M.; Niu, Z.; Wang, C.; Wu, C.; Wang, L. Use of MODIS and Landsat time series data to generate high-resolution temporal synthetic Landsat data using a spatial and temporal reflectance fusion model. J. Appl. Remote Sens. 2012, 6, 063507.

13. Zhang, W.; Li, A.; Jin, H.; Bian, J.; Zhang, Z.; Lei, G.; Qin, Z.; Huang, C. An enhanced spatial and temporal data fusion model for fusing Landsat and MODIS surface reflectance to generate high temporal Landsat-like data. Remote Sens. 2013, 5, 5346-5368. [CrossRef]

14. Huang, B.; Song, H. Spatiotemporal reflectance fusion via sparse representation. IEEE Trans. Geosci. Remote Sens. 2012, 50, 3707-3716. [CrossRef]

15. Song, H.; Huang, B. Spatiotemporal satellite image fusion through one-pair image learning. IEEE Trans. Geosci. Remote Sens. 2013, 51, 1883-1896. [CrossRef]

16. Loncan, L.; de Almeida, L.B.; Bioucas-Dias, J.M.; Briottet, X.; Chanussot, J.; Dobigeon, N.; Fabre, S.; Liao, W.; Licciardi, G.A.; Simoes, M.; et al. Hyperspectral pansharpening: A review. IEEE Geosci. Remote Sens. Mag. 2015, 3, 27-46. [CrossRef]

17. Vivone, G.; Alparone, L.; Chanussot, J.; Dalla Mura, M.; Garzelli, A.; Licciardi, G.A.; Restaino, R.; Wald, L. A critical comparison among pansharpening algorithms. IEEE Trans. Geosci. Remote Sens. 2015, 53, 2565-2586. [CrossRef]

18. Zhou, J.; Kwan, C.; Budavari, B. Hyperspectral image super-resolution: A hybrid color mapping approach. J. Appl. Remote Sens. 2016, 10, 035024. [CrossRef]

19. Memarsadeghi, N.; Mount, D.M.; Netanyahu, N.S.; Le Moigne, J. A fast implementation of the ISODATA clustering algorithm. Int. J. Comput. Geom. Appl. 2007, 17, 71-103. [CrossRef]

20. Planet Imagery Product Specifications. Available online: http://www.vekom.com/admin_area/kcfinder/ upload/files/Planet_Imagery_Product_Specs.pdf (accessed on 30 March 2018).

(C) 2018 by the authors. Licensee MDPI, Basel, Switzerland. This article is an open access article distributed under the terms and conditions of the Creative Commons Attribution (CC BY) license (http:/ / creativecommons.org/licenses/by/4.0/). 